\title{
MicroRNA-125b: association with disease activity and the treatment response of patients with early rheumatoid arthritis
}

Veronika Hruskova ${ }^{1,2^{*}}$, Romana Jandova ${ }^{1}$, Lucia Vernerova' ${ }^{1}$, Herman Mann ${ }^{1}$, Ondrej Pecha ${ }^{3}$, Klara Prajzlerova ${ }^{1}$, Karel Pavelka', Jiri Vencovsky ${ }^{1}$, Maria Filkova ${ }^{1}$ and Ladislav Senolt ${ }^{1}$

\begin{abstract}
Background: MicroRNAs (miRNAs) are small RNAs that regulate gene expression by targeting mRNA. It was proved that some miRNAs are significantly deregulated in rheumatoid arthritis (RA). MicroRNA-125b negatively regulates expression of TNF-a, which plays a crucial role in RA pathogenesis. The aim of this study was to determine the treatment outcome of patients with early RA based on the expression of circulating and cellular miR-125b.

Methods: Total RNA was isolated from the plasma and peripheral blood mononuclear cells (PBMCs) of 58 patients with early RA before and three months after treatment initiation and of 54 age- and sex-matched healthy controls (HC). The expression of miR-125b was measured by TaqMan quantitative PCR. The treatment responders were defined as patients achieving remission or low disease activity (28-joint count disease activity score (DAS28) <3.2). Receiver operating characteristic (ROC) curve and stepwise backward multivariable logistic regression analyses of miR-125b expression were used to predict the disease outcome at three and six months after initiation of treatment.
\end{abstract}

Results: The expression of miR-125b in the PBMCs and plasma of treatment-naive early RA patients was significantly lower than that of $\mathrm{HC}$ and increased significantly after three months of treatment, particularly in responders. However, only the cellular expression of miR-125b was inversely correlated with disease activity. MiR-125b expression in PBMCs was higher in responders than in non-responders after three months $(p=0.042)$. Using ROC analysis, the cellular expression of miR-125b, but not the disease activity at baseline, predicted the treatment response after three months of therapy (area under the curve 0.652 (95 \% Cl 0.510 to 0.793); $p=0.048$ ).

Conclusion: The expression of miR-125b in PBMCs of treatment-naive patients may present a novel biomarker for monitoring the treatment outcome during the early phase of RA.

Keywords: MicroRNA-125b, Early rheumatoid arthritis, Treatment outcome, Disease activity

\section{Background}

Rheumatoid arthritis (RA), which affects approximately $1 \%$ of the world population, is an autoimmune disease that if not treated effectively is associated with persistent synovitis, leading to severe joint destruction, development of joint deformities, and increased risk of cardiovascular diseases [1]. A targeted therapy approach is an

\footnotetext{
* Correspondence: hruskova@revma.cz

'Institute of Rheumatology and Department of Rheumatology, First Faculty of Medicine, Charles University in Prague, Na Slupi 4, 12850 Prague 2, Czech Republic

${ }^{2}$ Faculty of Science Charles University in Prague, Prague, Czech Republic

Full list of author information is available at the end of the article
}

optimal treatment strategy providing the best results for suppressing inflammation, thus avoiding irreversible joint damage and the development of comorbidities in patients with RA [2]. Some patients achieve remission or at least low disease activity shortly after the initiation of treatment with conventional synthetic disease-modifying antirheumatic drugs (cs-DMARDs), whereas others do not respond despite combination therapy or the use of biologic DMARDs. Despite tremendous progress in the treatment of RA, long-term remission in many patients and complete cure remain elusive $[3,4]$. 
One of the factors contributing to a lack of therapeutic response may be the epigenetic regulation of gene expression that seems to escape current targeted therapies [5]. Epigenetic modification is characterized by changes in gene expression without alteration of the nucleotide sequence. This process results from the posttranslational modification of DNA-binding molecules and from the posttranscriptional repression of targeted protein-coding genes [6]. The latter mechanism is mediated by a large number of small non-coding RNAs, including microRNA (miRNAs). MicroRNAs can degrade/destabilize targeted mRNA (gene interference) or inhibit protein synthesis and thereby regulate crucial pathways and cellular processes such as cell growth, differentiation, proliferation, and cell death $[7,8]$. Deregulation of some miRNAs has been found in many diseases $[9,10]$, including autoimmune inflammatory disorders such as RA [11-13]. There is evidence that miRNAs can be secreted extracellularly and can be present in plasma or serum in a stable form that is protected from endogenous RNase activity [14]. Furthermore, several circulating miRNAs have already been suggested as potential biomarkers of disease activity in RA $[15,16]$.

The identification of sensitive biomarkers that predict the response to therapy during the early phases of RA remains challenging. Recently, the circulating miR-125b levels have been associated with chemotherapeutic resistance in breast cancer patients [17], and an elevated serum level of miR-125b has been suggested to be a potential predictive biomarker of the treatment response to rituximab in patients with RA [18]. To date, no study has investigated the expression of miR-125b in patients with treatment-naïve early RA. Therefore, the aim of our study was to determine whether the expression of cellular or circulating miR-125b may predict the outcome of treatment in patients with early RA.

\section{Methods \\ Patients}

Fifty-eight patients with early RA who fulfilled the 2010 American College of Rheumatology (ACR)/European League Against Rheumatism (EULAR) classification criteria for RA [19], with a duration of symptoms $<6$ months were included in this study and were prospectively followed in the Prague Early RA Clinic (PERAC) at the Institute of Rheumatology, Prague, Czech Republic as previously described [20]. The control group consisted of 54 age-matched and sex-matched healthy individuals.

Disease activity was assessed using the 28-joint count disease activity score-erythrocyte sedimentation rate (DAS28-ESR) at baseline and at 3 and 6 months after the initiation of treatment. The patients were categorized into non-responders if they had moderate to high disease activity (DAS28 $\geq 3.2$ ) and into responders if they achieved low disease activity or remission after 3 or 6 months of treatment.

\section{RNA isolation}

Peripheral blood mononuclear cells (PBMCs) were isolated by standard Ficoll (Greiner Bio-one, Leipzig, Germany) density gradient centrifugation, and pellets were snap frozen and stored at $-80{ }^{\circ} \mathrm{C}$ until analysis. Plasma samples were stored at $-20{ }^{\circ} \mathrm{C}$. Total cellular RNA was isolated by miRNeasy Mini Kit (Qiagen, Hilden, Germany) according to protocol. Residual DNA contamination was removed using the RNase-Free DNase Set (Qiagen) as recommended by the manufacturer. Circulating miRNAs were isolated from plasma samples using phenol-chloroform extraction as previously described [16]. Plasma samples were spiked with 25 fmol each of 3 synthetic miRNAs of Caenorhabditis elegans origin (cel-miR-39, cel-miR-54, cel-miR-238) (Qiagen) after denaturation with Trizol LS (Thermo Scientific, Waltham, MA, USA) [16]. The concentration of total RNA was measured using NanoDrop 2000 spectrophotometer (Thermo Scientific).

\section{Reverse transcription and quantification of miRNAs}

The expression of miR-125b in PBMCs and plasma samples of all patients with early RA at baseline and at 3 months of therapy was analyzed and compared to the expression in PBMCs and plasma of HC. Overall, $5 \mathrm{ng}$ of total RNA was reverse-transcribed using TaqMan microRNA Assays (Life Technologies) and TaqMan MicroRNA Reverse Transcription Kit (Life Technologies) in a Thermocycler MyCycler (Biorad, Hercules, CA, USA). Next, TaqMan microRNA Assays (Life Technologies) and TaqMan Universal PCR Master Mix, no AmpErase UNG (Life Technologies) were used to quantify miRNA expression by quantitative polymerase chain reaction (qPCR) in a 7900HT Fast Real-Time PCR System (Applied Biosystems, Foster City, CA, USA). Small nucleolar RNA RNU44 for cellular miRNAs or the average of cel-miR-39, cel-miR-54 and cel-miR-238 for circulating miRNAs (Life Technologies) were used to normalize data in PBMCs or plasma samples, respectively. $X$-fold calculation using the delta cycle threshold $(\mathrm{dCt})$ method was used for calculating the relative expression of miR-125b as follows:

$$
\begin{aligned}
& (\text { Ct miR-125b-Ct RNU44/average of cel-miR-39, cel-miR-54, } \\
& 2^{-} \text {and cel-miR-238) }
\end{aligned}
$$

\section{Statistical analysis}

The Mann-Whitney $U$ test and Wilcoxon matched-pairs signed rank test were used where appropriate. Spearman's correlation test and the Fisher transformation were applied for analysis of correlation and partial correlation. Receiver operating characteristic (ROC) curve analysis of 
miR-125b expression was performed to predict disease outcome, and the area under the curve (AUC) with $95 \%$ confidence interval (CI) was calculated. To confirm the ROC analysis, stepwise backward multivariable logistic regression was performed. Data were presented as the mean (SD) or median (range). $P$ values $<0.05$ were considered statistically significant. GraphPad Prism, version 6 software was used for the statistical analyses.

\section{Results}

\section{Clinical characteristics}

The clinical characteristics of the patients are shown in Table 1. Prior to treatment, 37patients had high disease activity (DAS28 $>5.1$ ), 15 patients had moderate disease activity (DAS28 3.2-5.1), and 6 patients had low disease activity (DAS28 < 3.2). Treatment with cs-DMARDs was initiated in 56 patients at baseline: 49 patients were treated with methotrexate (mean weekly dose of $15 \mathrm{mg}$ at 3 months), 1 patient was treated with leflunomide, 4 patients were treated with sulfasalazine (mean daily dose of $2 \mathrm{~g}$ at 3 months), 2 patients were treated with antimalarial drugs (mean hydroxychloroquine dose $200 \mathrm{mg}$ ), 1 patient received a combination of methotrexate and sulfasalazine, and 46 patients received glucocorticoids (mean daily dose of $5 \mathrm{mg}$ of prednisone or equivalent at 3 months). Two patients were receiving only glucocorticoids at 3 months either due to liver toxicity (methotrexate was temporarily discontinued) or planned pregnancy (cs-DMARD was not initiated within the 3 months).
After 3 months of treatment, there was significant reduction in disease activity served (DAS28 decreased from $5.6 \pm$ 1.6 to $3.1 \pm 1.4$ and C-reactive protein (CRP) decreased from $9.2(0.3-152.2)$ to $3.1(0.2-23.9) \mathrm{mg} / \mathrm{l} ; p<0.001$ for all comparisons). After 3 months of treatment, 24 patients were in remission, 12 patients had low disease activity, 18 patients had moderate disease activity, and 4 patients had high disease activity. The improvement continued: after 6 months of treatment, 28 patients were in remission, 15 patients had low disease activity, 12 patients had moderate disease activity, and 3 patients had high disease activity.

\section{The expression of miR-125b is lower in patients with early RA}

First, we determined miR-125b expression in patients with early RA and in $\mathrm{HC}$ and the effect of treatment on the expression of miR-125b. The baseline expression of miR-125b in peripheral blood mononuclear cells (PBMCs) $(p=0.001)$ and in plasma $(p<0.0001)$ was significantly lower in patients with early RA than in $\mathrm{HC}$ and increased significantly after 3 months of therapy $(p=0.006$ and $p=$ 0.001 , respectively) (Fig. 1a, b). There was a significant increase in miR-125b expression in responders only, compared to patients who did not achieve remission or low disease activity (Fig. 1c, d). Similar changes in the miR$125 \mathrm{~b}$ expression profile were observed after 3 months in responders and non-responders after 6 months of treatment $(p=0.006$ and $p=0.001$, respectively). Increase in miR-125b expression over time was not associated with the dose of glucocorticoids or methotrexate. Furthermore,

Table 1 Characteristics of patients with early rheumatoid arthritis (RA) and healthy controls (HC)

\begin{tabular}{|c|c|c|c|c|}
\hline & Early RA & & & $\mathrm{HC}$ \\
\hline Number & 58 & & & 54 \\
\hline Sex, female/male, $n$ & $42 / 16$ & & & $41 / 13$ \\
\hline Age, years & $54.93 \pm 16.18$ & & & $50.87 \pm 15.11$ \\
\hline Disease duration & $<6$ months & & & \\
\hline \multicolumn{5}{|l|}{ Treatment after 3 months } \\
\hline Glucocorticoids & 46 & & & \\
\hline Methotrexate & 49 & & & \\
\hline Leflunomide & 1 & & & \\
\hline Sulfasalazine & 4 & & & \\
\hline Hydroxychloroquine & 2 & & & \\
\hline Clinical characteristic & Baseline & 3 months & 6 months & \\
\hline RF IgM positivity, \% & 52 & NA & NA & NA \\
\hline Anti-CCP positivity, $\%$ & 66 & NA & NA & NA \\
\hline DAS28 & $5.56 \pm 1.56$ & $3.05 \pm 1.42$ & $2.86 \pm 1.25$ & NA \\
\hline CRP, mg/l & $9.16(0.30,152.20)$ & $3.10(0.21,23.85)$ & $2.83(0.24,44.44)$ & $1.02(0.22,4.36)$ \\
\hline $\mathrm{ESR}, \mathrm{mm} / \mathrm{h}$ & $27.00(4.00,107.00)$ & $15.50(2.00,54.00)$ & $14.00(2.00,74.00)$ & NA \\
\hline
\end{tabular}

Data are expressed as the mean \pm SD or median (range) according to the distribution. Anti-CCP anti-citrullinated antibodies, CRP C-reactive protein, DAS28 disease activity score, ESR erythrocyte sedimentation rate, $H C$ healthy controls, NA not analyzed, RF rheumatoid factor 


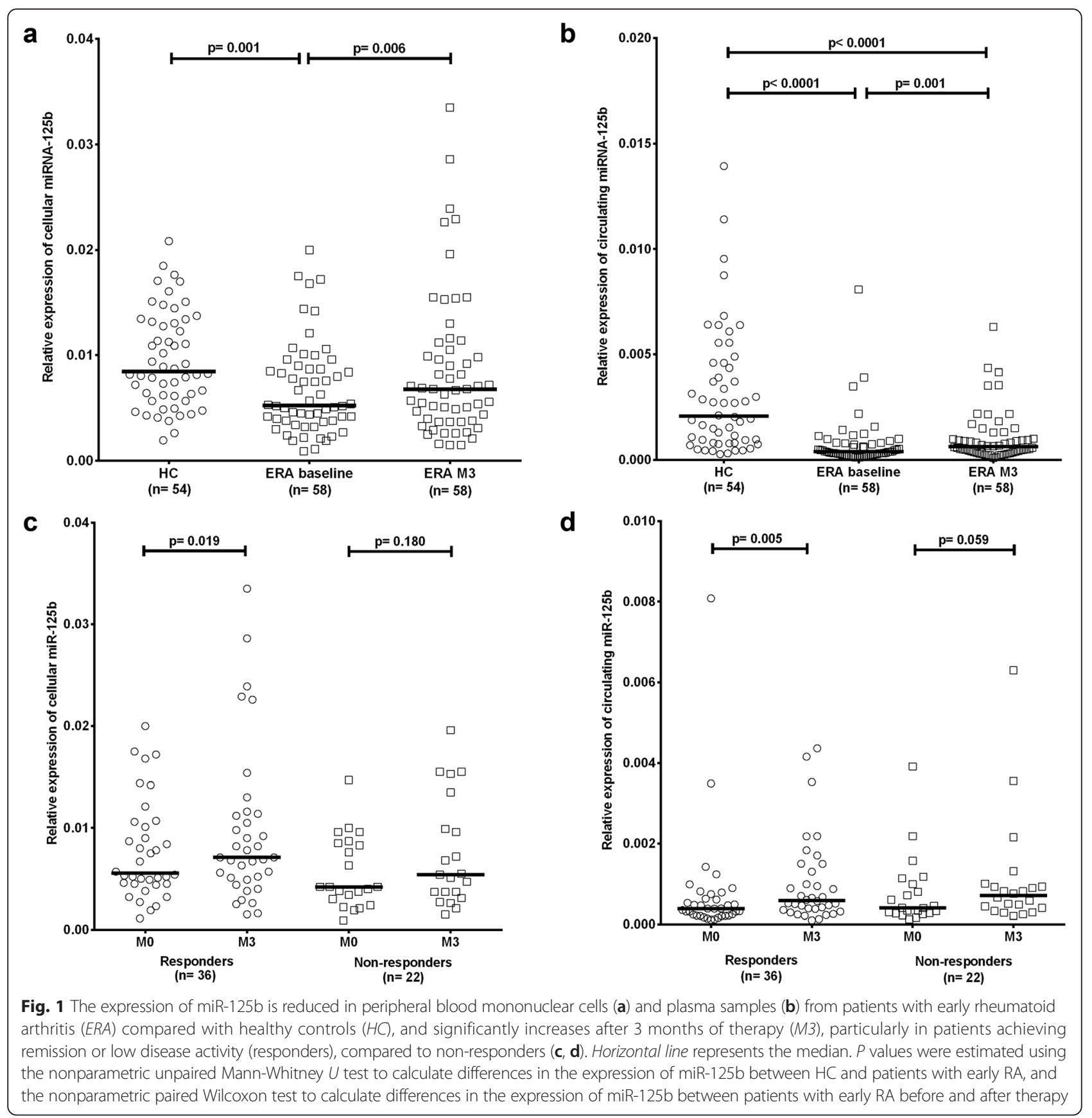

miR-125b expression in PBMCs also increased in the few responders who were naïve to glucocorticoids (data not shown). However, there was no association between cellular and circulating miR-125b $(r=-0.222, p=0.094)$ and no association between the change in cellular or circulating miR-125b and an improvement in disease activity (data not shown).

Cellular miR-125b is inversely correlated with disease activity Next, we analyzed whether the expression of miR-125b is associated with disease activity in patients with early
RA. We found that the baseline expression of cellular miR-125b was inversely correlated with the DAS28 at baseline $(r=-0.407 ; p=0.001)$ (Fig. 2), with baseline ESR $(r=-0.375 ; p=0.003)$ and with CRP levels $(r=-0.270$; $p=0.035)$. Using age-adjusted analysis, the inverse correlation between miR-125b and disease activity remained significant for DAS28 $(r=-0.276 ; p=0.032)$ and ESR $(r=-0.268 ; p=0.042)$ but not for CRP levels $(r=-0.194 ; p=0.137)$. No such correlations were demonstrated for circulating miR-125b $(r=0.117, p=0.384)$. These data show that lower intracellular expression of 


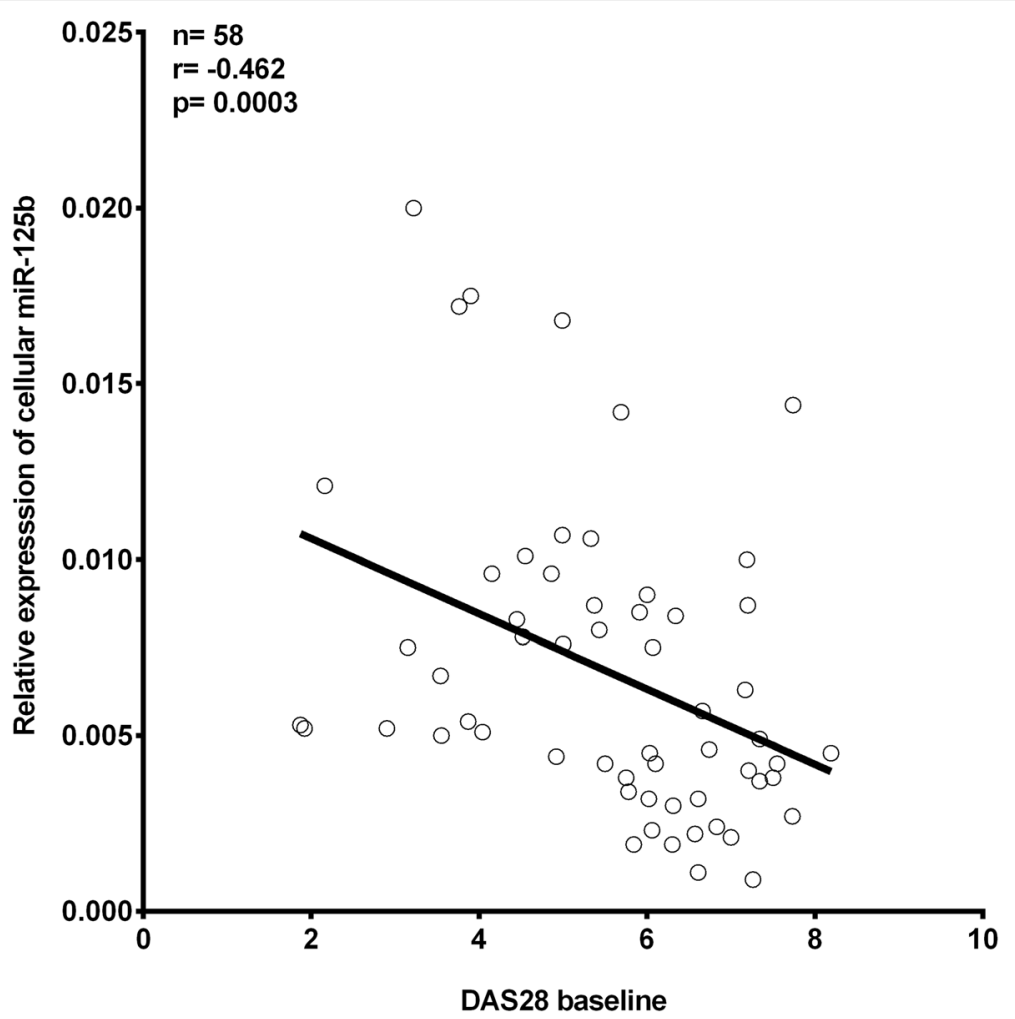

Fig. 2 Inverse correlation between the initial expression of miR-125b in peripheral blood mononuclear cells and baseline disease activity assessed by the 28-joint count disease activity score (DAS28). Nonparametric Spearman correlation analysis was used to test the association between baseline miR-125b and disease activity

miR-125b in PBMCs is present in treatment-naïve patients with early RA, who have higher disease activity.

\section{Baseline expression of cellular miR-125b predicts treatment response}

We also hypothesized that baseline expression of miR$125 \mathrm{~b}$ may predict treatment response. We demonstrated that baseline cellular (but not circulating) expression of miR-125b was higher in responders to treatment at 3 months than in non-responders $(p=0.042)$ (Fig. 3). However, baseline miR-125b expression did not differ between responders and non-responders to treatment at the 6-month follow up $(p=0.321)$, probably due to the small number of non-responders $(n=15)$.

Based on the data showing that baseline miR-125b expression is higher in responders than in non-responders at 3 months, we performed ROC curve analysis to determine the predictive value of baseline miR-125b expression and conventional baseline parameters of disease activity, including DAS28, CRP levels and ESR, in separating patients with early RA who achieve an optimal treatment outcome (remission or low disease activity) from those who do not have such a response. This analysis showed that baseline miR-125b expression in PBMCs was the only predictor of achieving an optimal treatment outcome after
3 months (Fig. 4). The AUC for baseline miR-125b expression was 0.663 (95\% CI 0.520 to $0.805 ; p=0.048$ ). We then performed stepwise backward multivariate logistic regression analysis of baseline cellular miR-125b expression in combination with DAS28 at 3 months. This analysis confirmed the ROC analysis outcomes and showed that baseline miR-125b expression was a significant and independent predictor of treatment response at 3 months (OR $3.71795 \%$ CI 1.005 to $13.745 ; p=0.049$ ).

\section{Discussion}

In the present study, we report that (1) baseline miR$125 \mathrm{~b}$ expression is lower in patients with early RA than in healthy subjects, (2) cellular miR-125b expression is inversely correlated with RA disease activity, (3) miR$125 \mathrm{~b}$ expression increased after 3 months of conventional therapy, and (4) higher baseline cellular miR-125b expression predicts the early optimal therapeutic response (DAS28 < 3.2).

The expression of several miRNAs is altered in patients with RA compared to the healthy population [21]. Consistent with previous studies demonstrating reduced expression of miR-125b in some autoimmune diseases, particularly in psoriatic keratinocytes and systemic lupus erythematosus (SLE) CD4+ T lymphocytes [22, 23], we 


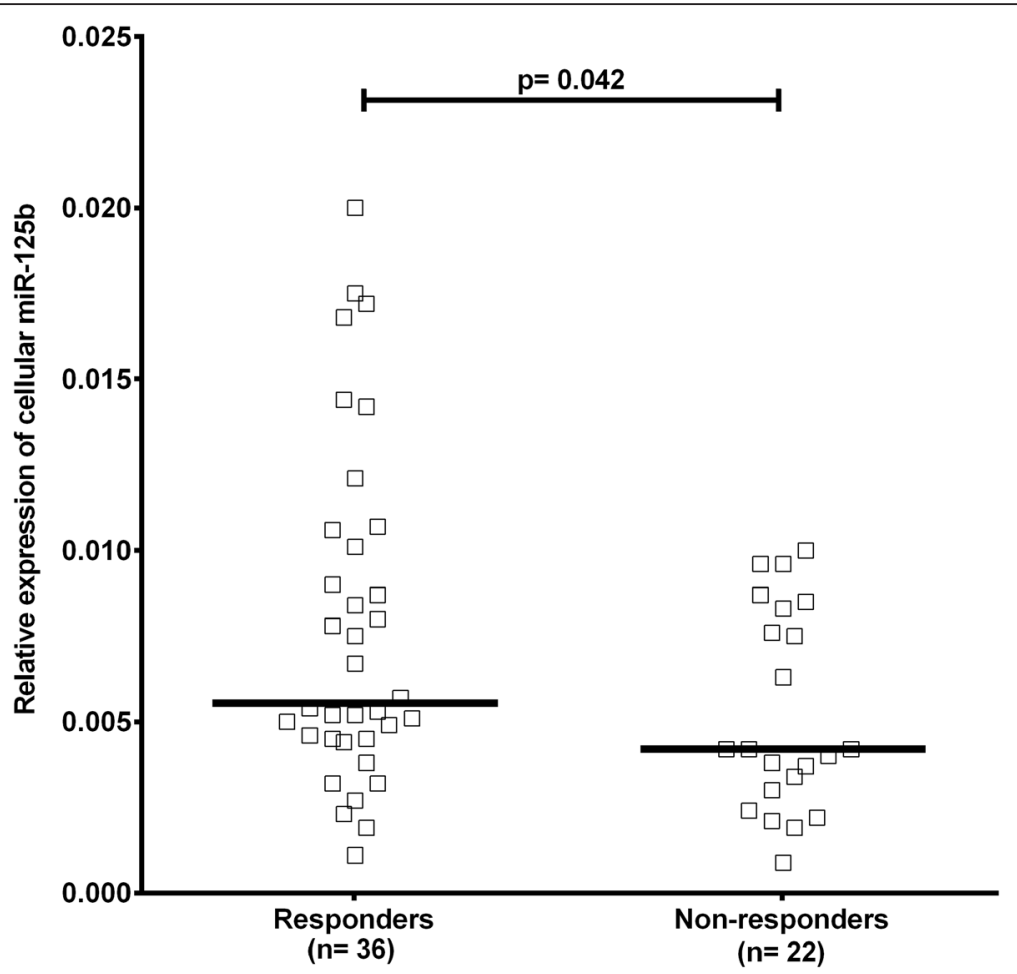

Fig. 3 Baseline expression of miR-125b in peripheral blood mononuclear cells is higher in responders than in non-responders. Patients with early rheumatoid arthritis were categorized as non-responders if they had moderate to high disease activity assessed by the 28-joint count disease activity score (DAS28 2 3.2) and as responders if they achieved low disease activity or remission (DAS28 < 3.2) after 3 months of treatment. Horizontal line represents the median. $P$ values were estimated using the nonparametric unpaired Mann-Whitney $U$ test to calculate differences in the expression of miR-125b between responders and non-responders

found that the expression of miR-125b in both PBMCs and plasma is lower in treatment-naïve patients with early RA than in HC.

MiR-125b has been described as a negative regulator of TNF $\alpha$ and other pro-inflammatory cytokines such as interferon (IFN) $\gamma$, chemokine CCL4 and matrix metalloproteinase (MMP)-13 [24-27]. Thus, we hypothesize that reduced expression of miR-125b could be associated with increased inflammation in RA. However, Duroux-Richard et al. [18] reported increased expression of miR-125b in whole blood and serum from patients with established RA. The discrepancy between this report [18] and our data may be explained by the different material used for miRNA analysis and particularly by the different stages of the disease. It was previously shown that the expression profile of miRNAs is different in whole blood and PBMCs [28]. Moreover, although our study included treatmentnaive patients with RA of short disease duration, DurouxRichard et al. [18] studied patients with established and long-lasting RA, consistent with our recent study that showed differential expression of circulating miRNAs in early and in established RA [16].

We observed inverse correlation between baseline expression of cellular miR-125b and the parameters of disease activity in patients with early RA. This result is consistent with an inhibitory effect of miR-125b on the expression of pro-inflammatory cytokines, cell proliferation, and apoptosis [24-27, 29]. miR-125b has been inversely associated with age, as its expression is higher in the immune cells of young donors than in older donors [29]. Considering age as a potential confounder, age-adjustment confirmed our data, and the association between baseline miR-125b expression in PBMCs and clinical disease activity remained significant. Furthermore, we demonstrated upregulation of miR-125b in PBMCs from patients with early RA after 3 months of therapy, which was particularly pronounced in responders. This finding may be due to stronger inhibition of pro-inflammatory cytokines, which would lead to a better clinical outcome over time. This hypothesis is supported by a recent observation of increased levels of circulating miR-125b in RA responders to anti-TNF $\alpha / D M A R D$ combination therapy [30], though the source of miRNA was not cellular.

Although one study demonstrated upregulation of miR-125b in human B lymphoblast cells after exposure to dexamethasone [31], this upregulation was transient. In our study, we found no association between the dose of glucocorticoids and change in miR-125b expression over time. Furthermore, miR-125b expression in PBMCs also increased in the few responders who were naïve to 


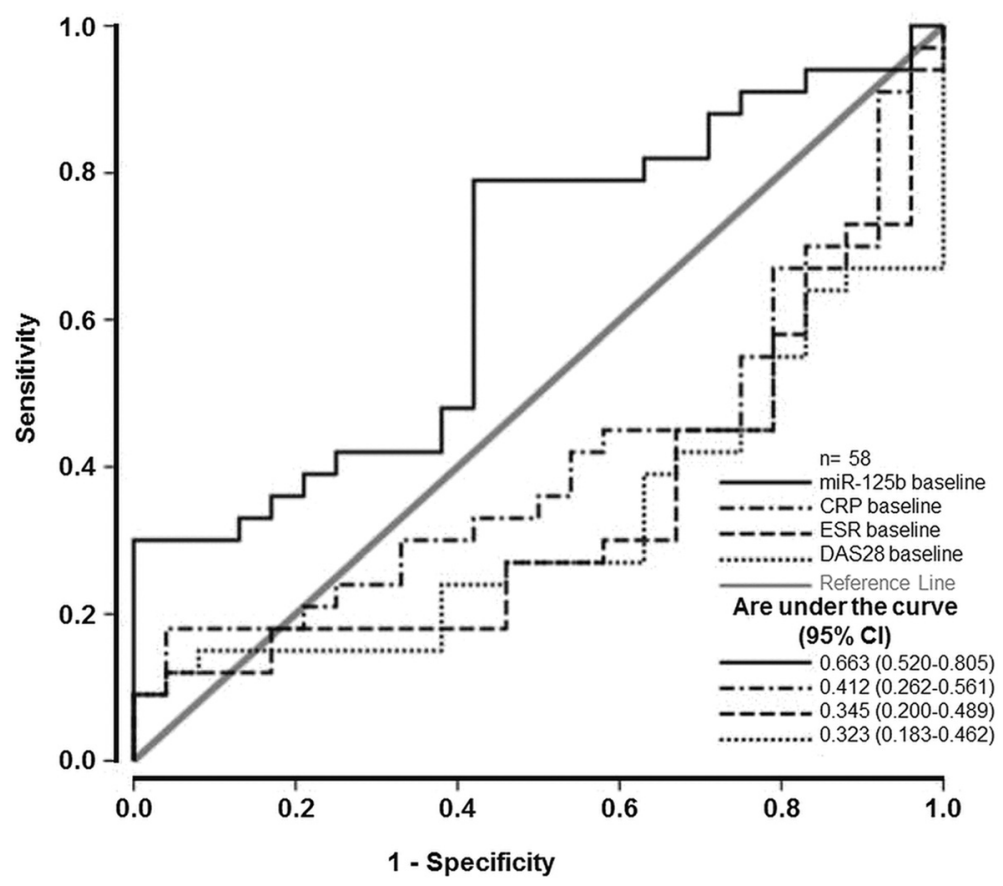

Fig. 4 Receiver operating characteristic curve analysis of baseline expression of miR-125b in peripheral blood mononuclear cells as a predictor of achieving optimal treatment outcome (28-joint count disease activity score (DAS28<3.2) after 3 months. Baseline miR-125b expression (solid black line) had the highest area under the curve (0.663 (0.520-0.805); $p=0.048)$. C-reactive protein (CRP) (dashed-dotted black line), erythrocyte sedimentation rate (ESR) (dashed black line) and DAS28 (dotted black line) at baseline are presented

glucocorticoids. We also did not observe association between the dose of methotrexate and change in miR-125b over time, therefore, it can be speculated that the change in miR-125b expression may not be influenced by the type of therapy, but rather by improvement in disease activity.

To determine the treatment outcome, we performed a predictive analysis by plotting an ROC curve and by logistic regression analysis of the baseline expression of miR-125b and disease activity over time. We demonstrated that higher baseline expression of miR-125b predicts remission or low disease activity after 3 months of therapy. Crucial pro-inflammatory cytokines such as TNF $\alpha$ or IL-6 [24-27, 32] are known downstream targets of miR125. Therefore, we suggest that the higher cellular miR125b expression contributes to the achievement of lower inflammatory status and thus, could serve as a biomarker for the early treatment response in patients with early RA. However, no such finding was observed for circulating miR-125b or for miR-125b in PBMCs at the 6-month follow up. We found no correlation between circulating and miR-125b containing PBMCs; therefore, other compartments or mechanisms may influence the levels of circulating miRNAs. Because miR-125b has been demonstrated to be a biomarker of resistance to chemotherapy in pancreatic cancer [32] and circulating miR-125b has been demonstrated to be a predictive biomarker of response to biologic treatment in established RA $[18,30]$, further studies evaluating the role of miR-125b in RA are needed.

\section{Conclusion}

In conclusion, we have demonstrated that the expression of miR-125b in PBMCs is lower in treatment-naïve patients with early RA and mainly in patients who do not reach the optimal therapeutic outcome at 3-month follow-up. The expression of cellular, but not circulating, miR-125b is inversely associated with RA disease activity and may serve as a potential biomarker of treatment response in early RA.

\section{Abbreviation}

ACR, American College of Rheumatology; Anti-CCP, anti-citrullinated antibodies; AUC, area under the curve; CCL4, C-C motif chemokine ligand 4; Cl, confidence interval; CRP, C-reactive protein; Cs-DMARDs, conventional synthetic disease-modifying antirheumatic drugs; DAS28, 28-joint count disease activity score; DMARDs, disease modifying antirheumatic drugs; ESR, erythrocyte sedimentation rate; EULAR, European League Against Rheumatism; HC, healthy controls; IFNy, interferon gamma; IL-6, interleukin 6; miR, miRNA, microRNA; MMP-13, matrix metalloproteinase 13; NA, not analyzed; OR, odds ratio; PBMCs, peripheral blood mononuclear cells; PCR, polymerase chain reaction; PERAC, Prague Early RA Clinic; $\mathrm{QPCR}$, quantitative polymerase chain reaction; RA, rheumatoid arthritis; RF, rheumatoid factor; ROC, receiver operating characteristic curve; SD, standard deviation; SLE, systemic lupus erythematosus; TNFa, tumor necrosis factor alpha

\section{Acknowledgements}

We thank all the rheumatologists and patients involved in the study for their participation. The dataset supporting the conclusions of this article is included within the article. This work was supported by IGA project No. NT 
14498, by project No. 023728 for the conceptual development of research organization, by the Ministry of Health, Czech Republic and by GAUK-367615 Charles University in Prague, Czech Republic and Research Project SW 260263.

\section{Authors' contributions}

VH made substantial contributions to data acquisition, analysis, and interpretation, and drafted the manuscript. RJ and KIP made substantial contributions to data acquisition and analysis, and revised the manuscript critically. LV and OP made substantial contributions to statistical analysis and interpretation of the data and helped to revise the manuscript critically. HM made substantial contributions to data acquisition and revised the manuscript critically. KP, JV, and MF revised the manuscript critically for important intellectual content. LS made substantial contributions to study design, and helped with data analysis and interpretation, and drafting of the manuscript. All authors read and approved the final manuscript.

\section{Competing interests}

The authors declare that they have no competing interests.

\section{Author details}

${ }^{1}$ Institute of Rheumatology and Department of Rheumatology, First Faculty of Medicine, Charles University in Prague, Na Slupi 4, 12850 Prague 2, Czech Republic. ${ }^{2}$ Faculty of Science Charles University in Prague, Prague, Czech Republic. ${ }^{3}$ Technology Centre ASCR, Prague, Czech Republic.

\section{Received: 7 April 2016 Accepted: 17 May 2016}

Published online: 02 June 2016

\section{References}

1. Mclnnes IB, O'Dell JR. State-of-the-art: rheumatoid arthritis. Ann Rheum Dis. 2010;69:1898-906

2. Stoffer MA, Schoels MM, Smolen JS, Aletaha D, Breedveld FC, Burmester G, et al. Evidence for treating rheumatoid arthritis to target: results of a systematic literature search update. Ann Rheum Dis. 2016;75:16-22.

3. van Eijk IC, Nielen MM, van der Horst-Bruinsma I, Tijhuis GJ, Boers M Dijkmans BA, et al. Aggressive therapy in patients with early arthritis results in similar outcome compared with conventional care: the STREAM randomized trial. Rheumatology (Oxford). 2012;51:686-94.

4. Hobbs KF, Cohen MD. Rheumatoid arthritis disease measurement: a new old idea. Rheumatology (Oxford). 2012;51 Suppl 6:vi21-7.

5. Migliore C, Giordano S. Resistance to targeted therapies: a role for microRNAs? Trends Mol Med. 2013:19:633-42.

6. Jüngel A, Ospelt C, Gay S. What can we learn from epigenetics in the year 2009? Curr Opin Rheumatol. 2010;22:284-92.

7. Bartel DP. MicroRNAs: target recognition and regulatory functions. Cell. 2009:136:215-33.

8. O'Connell RM, Rao DS, Baltimore D. microRNA regulation of inflammatory responses. Annu Rev Immunol. 2012;30:295-312.

9. Lujambio A, Lowe SW. The microcosmos if cancer. Nature. 2012:482:347-55.

10. Romaine SPR, Tomaszewski M, Condorelli G, Samani NJ. MicroRNAs in cardiovascular disease: an introduction for clinicians. Heart. 2015;101:921-8.

11. Duroux-Richard I, Jorgensen C, Apparailly F. What do microRNAs mean for rheumatoid arthritis? Arthritis Rheum. 2012:64:11-20.

12. Baxter D, Mclnnes IB, Kurowska-Stolarska M. Novel regulatory mechanisms in inflammatory arthritis: a role for microRNA. Immunol Cell Biol. 2012;90:288-92.

13. Filková M, Jüngel A, Gay RE, Gay S. MicroRNAs in rheumatoid arthritis: potential role in diagnosis and therapy. BioDrugs. 2012:26:131-41.

14. Mitchell PS, Parkin RK, Kroh EM, Fritz BR, Wyman SK, Pogosova-Agadjanyan EL, et al. Circulating microRNAs as stable blood-based markers for cancer detection. Proc Natl Acad Sci USA. 2008;105:10513-8.

15. Murata K, Yoshitomi H, Tanida S, Ishikawa M, Nishitani K, Ito H, et al. Plasma and synovial fluid microRNAs as potential biomarkers of rheumatoid arthritis and osteoarthritis. Arthritis Res Ther. 2010;12:R86

16. Filková M, Aradi B, Senolt L, Ospelt C, Vettori S, Mann H, et al. Association of circulating miR-223 and miR-16 with disease activity in patients with early rheumatoid arthritis. Ann Rheum Dis. 2014;73:1898-904.

17. Wang H, Tan G, Dong L, Cheng L, Li K, Wang Z, et al. Circulating MiR-125b as a marker predicting chemoresistance in breast cancer. PLoS One. 2012;7:e34210.
18. Duroux-Richard I, Pers YM, Fabre S, Ammari M, Baeten D, Cartron G, et al. Circulating miR-125b is a potential biomarker predicting response to rituximab in rheumatoid arthritis. Mediators Inflamm. 2014;2014:342524.

19. Aletaha D, Neogi T, Silman AJ, Funovits J, Felson DT, Bingham 3rd CO, et al. 2010 rheumatoid arthritis classification criteria: an American College of Rheumatology/European League Against Rheumatism collaborative initiative. Ann Rheum Dis. 2010;69:1580-8.

20. Sglunda O, Mann H, Hulejová H, Kuklová M, Pecha O, Pleštilová L, et al. Decreased circulating visfatin is associated with improved disease activity in early rheumatoid arthritis: data from the PERAC cohort. PLOS One. 2014:9:e103495.

21. Churov AV, Oleinik EK, Knip M. MicroRNAs in rheumatoid arthritis: Altered expression and diagnostic potential. Autoimmun Rev. 2015;14:1029-37.

22. Xu N, Brodin P, Wei T, Meisgen F, Eidsmo L, Nagy N, et al. MiR-125b, a MicroRNA downregulated in psoriasis, modulates keratinocyte proliferation by targeting FGFR2. J Invest Dermatol. 2011;131:1521-9.

23. Luo X, Zhang L, Li M, Zhang W, Leng X, Zhang F, et al. The role of miR-125b in T lymphocytes in the pathogenesis of systemic lupus erythematosus. Clin Exp Rheumatol. 2013;31:263-71.

24. Tili E, Michaille J, Cimino A, Costinean S, Dumitru CD, Adair B, et al. Modulation of miR-155 and miR-125b levels following lipopolysacharide/ TNFa stimulation and their possible roles in regulating the response to endotoxin shock. J Immunol. 2007;179:5082-9.

25. Rajaram MV, Ni B, Morris JD, Brooks MN, Carlson TK, Bakthavachalu B, et al. Mycobacterium tuberculosis lipomannan blocks TNF biosynthesis by regulating macrophage MAPK-activated protein kinase 2 (MK2) and microRNA miR-125b. Proc Natl Acad Sci USA. 2011;108:17408-13.

26. Huang HC, Yu HR, Huang LT, Huang HC, Chen RF, Lin IC, et al. miR-125b regulates TNF- production in CD14+ neonatal monocytes via post-transcriptional regulation. J Leukoc Biol. 2012;92:171-82.

27. Rossi RL, Rossetti G, Wenandy L, Curti S, Ripamonti A, Bonnal RJ, et al. Distinct microRNA signatures in human lymphocyte subsets and enforcement of the naive state in CD4+ T cells by the microRNA miR-125b. Nat Immunol. 2011;12:796-803.

28. Atarod S, Smith H, Dickinson A, Wang XN. Important considerations for microRNA extraction methods from whole blood and peripheral blood mononuclear cells. F1000Res. 2014:3:183.

29. Cheng NL, Chen X, Kim J, Shi AH, Nguyen C, Wersto R, et al. MicroRNA-125b modulates inflammatory chemokine CCL4 expression in immune cells and its reduction causes CCL4 increase with age. Aging Cell. 2015;14:200-8.

30. Castro-Villegas C, Pérez-Sánchez C, Escudero A, Filipescu I, Verdu M, Ruiz-Limón $\mathrm{P}$, et al. Circulating miRNAs as potential biomarkers of therapy effectiveness in rheumatoid arthritis patients treated with anti-TNFa. Arthritis Res Ther. 2015:17:49.

31. Murray MY, Rushworth SA, Zaitseva L, Bowles KM, MacEwan DJ. Attenuation of dexamethasone-induced cell death in multiple myeloma is mediated by miR-125b expression. Cell Cycle. 2013;12:2144-53.

32. Yin $H$, Sun $Y$, Wang $X$, Park J, Zhang $Y$, Li M, et al. Progress on the relationship between miR-125 family and tumorigenesis. Exp Cell Res. 2015;339:252-60.

\section{Submit your next manuscript to BioMed Central and we will help you at every step:}

- We accept pre-submission inquiries

- Our selector tool helps you to find the most relevant journal

- We provide round the clock customer support

- Convenient online submission

- Thorough peer review

- Inclusion in PubMed and all major indexing services

- Maximum visibility for your research

Submit your manuscript at www.biomedcentral.com/submit
) Biomed Central 